\title{
Reply to Letter to the Editor to "Soy isoflavone intake and prevalence of depressive symptoms during pregnancy in Japan: baseline data from the Kyushu Okinawa Maternal and Child Health Study"
}

\author{
Yoshihiro Miyake $^{1,2} \cdot$ Keiko Tanaka $^{1,2} \cdot$ Hitomi Okubo $^{3} \cdot$ Satoshi Sasaki $^{4}$. \\ Shinya Furukawa ${ }^{1,2} \cdot$ Masashi Arakawa $^{5}$
}

Published online: 12 April 2017

(C) Springer-Verlag Berlin Heidelberg 2017

We thank Drs Abbas Smiley, Jennifer Cullin, Elizabeth Kaschalk, Ka He for their interest in our recent paper [1] published in European Journal of Nutrition. We have not yet investigated the relationships between sleep and physical activity and the prevalence of depressive symptoms during pregnancy. According to this comment, additional adjustment was made for sleep disturbance including the latency of falling asleep, continuity of night sleep, or early morning awakening (the prevalence: 16.8\%) and leisuretime physical activity at least once a week (the prevalence: $32.8 \%$ ). The additional adjusted prevalence ratio between extreme quartiles for total soy products was 0.64 (95\% CI $0.47-0.85$; $P$ for trend=0.002). Again, we express our appreciation for their comment.

This reply refers to letter to the editor available at doi:10.1007/ s00394-017-1428-9.

This reply refers to the original article available at doi:10.1007/ s00394-016-1327-5.

Yoshihiro Miyake

miyake.yoshihiro.ls@ehime-u.ac.jp

1 Department of Epidemiology and Preventive Medicine, Ehime University Graduate School of Medicine, Ehime 791-0295, Japan

2 Epidemiology and Medical Statistics Unit, Translational Research Center, Ehime University Hospital, Ehime, Japan

3 Department of Health Promotion, National Institute of Public Health, Saitama, Japan

4 Department of Social and Preventive Epidemiology, School of Public Health, The University of Tokyo, Tokyo, Japan

5 Health Tourism Research Fields, Graduate School of Tourism Sciences, University of the Ryukyus, Okinawa, Japan

\section{Reference}

1. Miyake Y, Tanaka K, Okubo H, Sasaki S, Furukawa S, Arakawa M (2016) Soy isoflavone intake and prevalence of depressive symptoms during pregnancy in Japan: baseline data from the Kyushu Okinawa Maternal and Child Health Study. Eur J Nutr 